Volume 8, No.6, November - December 2019

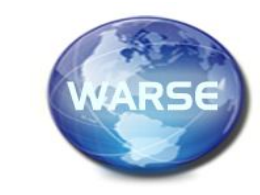

International Journal of Advanced Trends in Computer Science and Engineering

Available Online at http://www.warse.org/IJATCSE/static/pdf/file/ijatcse119862019.pdf

https://doi.org/10.30534/ijatcse/2019/119862019

\title{
Contribution to development of a new transformer with a single magnetic shunt for industrial microwave generators
}

\author{
Rajaa Oumghar $^{1}$, Mohammed Chraygane ${ }^{2}$, Hamid Outzguinrimt ${ }^{3}$, Mouhcine Lahame ${ }^{4}$, Ali Bouzit ${ }^{5}$, \\ ${ }^{1}$ ESTA-MSTI Research Team, ENSA-Ibn Zohr University, Morocco, raja.oumghar@edu.uiz.ac.ma \\ ${ }^{2}$ ESTA-MSTI Research Team-Ibn Zohr University, Morocco, m.chraygane@uiz.ac.ma \\ ${ }^{3}$ ESTA-MSTI Research Team, ENSA-Ibn Zohr University, Morocco, hamid.outzguinrimt@edu.uiz.ac.ma \\ ${ }^{4}$ ESTA-MSTI Research Team, ENSA--Ibn Zohr University, Morocco, mouhcine.lahame@edu.uiz.ac.ma \\ ${ }^{5}$ IRF-SIC Laboratory, Ibn Zohr University, Morocco, a.bouzit@uiz.ac.ma
}

\begin{abstract}
The main objective of this work is to treat the different proposed geometrical configurations of a high voltage transformer with a single magnetic shunt instead of that of dual magnetic shunts for industrial microwave generators power supply. This article deals with development of the single-phase high-voltage power supply based on a new model of equivalent column-type magnetic circuit for a special transformer with a single magnetic shunt, which has a special structure and wide applications. This work shows a detailed design and implementation procedure, including the design of the power supply. The sizing and the feasibility study of the equivalent transformer is covered in this article
\end{abstract}

The experimental results are provided in order to validate the model proposed. The electric diagram obtained of this model is tested in nominal mode, using the software EMTP (Electro-Magnetic Transients Program). The electrical operation simulation was consistent with the experiments in the case of conventional power supply.

Key words: microwave, Power supply, magnetron, equivalent transformer, magnetic shunt, geometrical configuration, feasibility study, modeling, simulation.

\section{INTRODUCTION}

This paper is part of the study of the high voltage HV single-phase power supply for microwave generators intended for industrial, scientific and medical applications [1-2-13]. The magnetron is a gridless vaccum tube that produces microwave energy. The characteristic of the magnetron imposes conditions to respect in order to ensure its good nominal functioning, namely current stabilization and limitation at the power supply [4-7]. The main part of this magnetron power supply, which ensures the conditions imposed, is its special transformer with double magnetic shunt. It provides in its secondary an average alterative tension of 2300 volts.

Then the secondary output of the transformer is rectified and doubled to a continuous voltage of $4000 \mathrm{~V}$ using a voltage doubler circuit, which consisting of a diode and a capacitor HV to power the magnetron [6].

The research carried out on the current supply helped to find a geometric equivalence of its single-phase high-voltage transformer [1-2-8-10]. So, the equivalent transformer with a single magnetic shunt (TSMS) can replace the conventional transformer with dual magnetic shunt (TDMS). In this sense, the work presented in this paper is to improve the new HV TSMS equivalent to the usual TDMS. We propose in the first part a detailed study of possible and feasible geometrical configurations, based on the different physical parameters involved in the expression of the reluctance of each part of the transformer. After dimensioning and verification of the constraints of realization for each geometric configuration, we proceed to modeling and simulation with EMTP of the new power supply based on the equivalent transformer.

\section{SIZING AND FEASIBILITY STUDY OF THE EQUIVALENT TRANSFORMER}

\subsection{Presentation of the equivalent transformer}

The basis of this work is to design and model the new power supply of a magnetron using equivalent transformer with a single magnetic shunt as shown in figure. 1

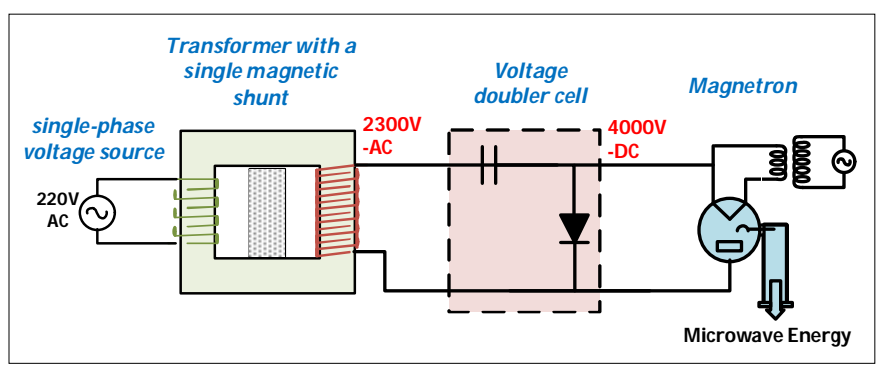

Figure 1: The circuit of the new power supply for a magnetron. 
The study done on the usual High Voltage HV Transformer with Dual Magnetic Shunts (TDMS) [3-154], in the figure. 2, shows that this one is equivalent to the new HV tranformer with a single magnetic shunt TSMS, in figure. 4 [1-2-9]. The cores and yokes of the latter have the same section and its magnetic circuit is geometrically symmetrical with respect to the equivalent shunt.

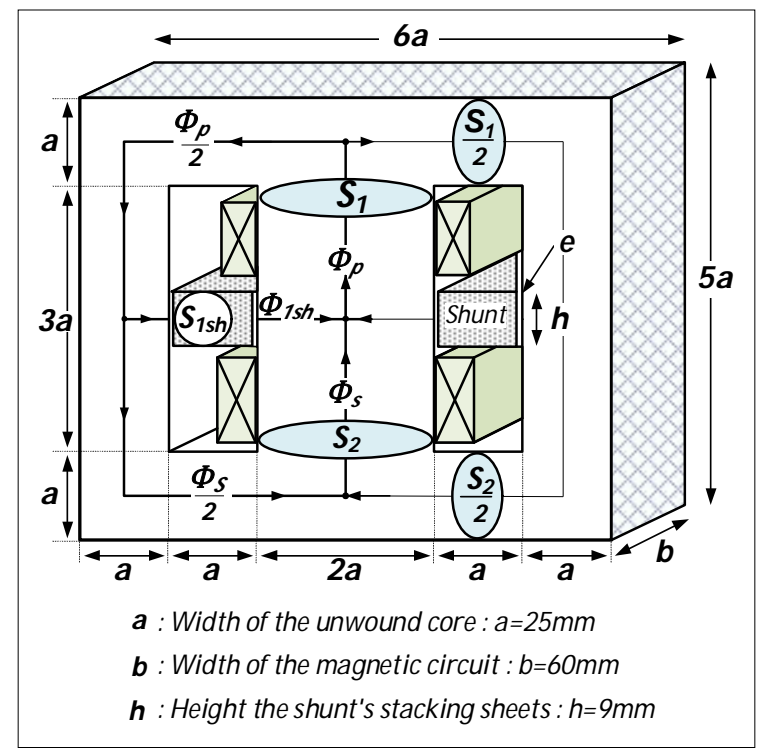

Figure 2: Structure and geometric parameters of the TDMS

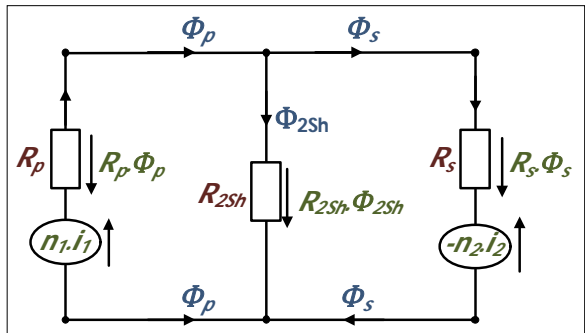

Figure 3: Equivalent magnetic diagram of the transformer

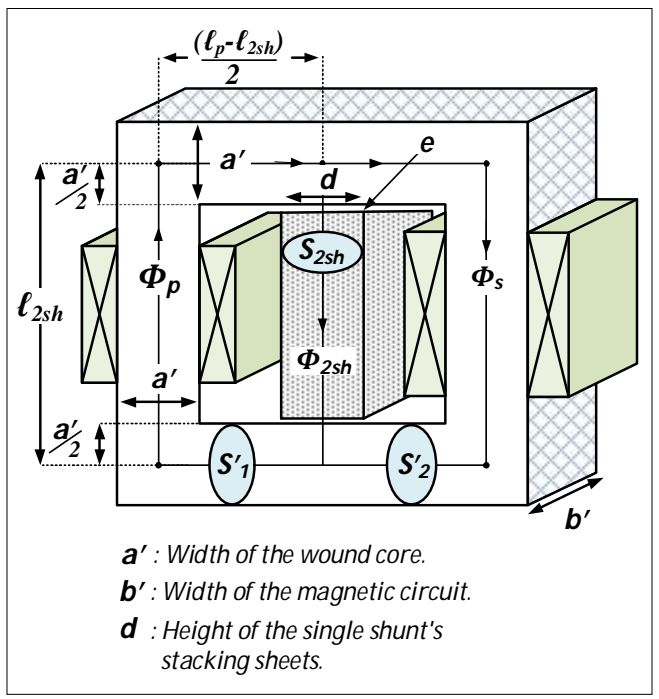

Figure 4: Structure and geometric parameters of the equivalent TSMS
The modeling of the ordinary transformer is no longer valid for transformers with magnetic shunts [5-8-13-15]. Indeed, The study of the TDMS allowed to describe its operation by the following system of magnetic and electric equations [2-10-11-12-19]:

$$
\left\{\begin{array}{l}
u_{1}=r_{1} \cdot i_{1}+n_{1} \cdot \frac{d \phi_{p}}{d t} \\
u_{2}=-r_{2} \cdot i_{2}+n_{2} \cdot \frac{d \phi_{S}}{d t} \\
R_{p} \cdot \phi_{p}+R_{1 S h} \cdot \phi_{1 S h}=n_{1} \cdot i_{1} \\
R_{S} \cdot \phi_{S}-R_{1 S h} \cdot \phi_{1 S h}=-n_{2} \cdot i_{2} \\
\phi_{P}=\phi_{2 S h}+\phi_{S}
\end{array}\right.
$$

The magnetic diagram with only one magnetic shunt of figure. 3 responds also to the preceding system of equations. This makes it possible to replace the magnetic circuit with two shunts of the usual TDMS by a new equivalent circuit with a single magnetic shunt. We thus find the new geometric design of the equivalent transformer with a single magnetic shunt (TSMS) of figure. 4.

With:

- $u_{1}, u_{2}$ : Voltage of the primary and secondary circuit.

- $r_{1}, r_{2}$ : Resistance of the primary and secondary winding.

- $i_{1}, i_{2}$ : Current flowing in the primary and secondary winding.

- $n_{1}, n_{2}$ : Number of the primary and secondary winding turns: $n_{1}=224, n_{2}=2400$.

- $R_{P}$ : Reluctance of the primary circuit, length $\ell_{P}=6,5 . a$, section $S_{1}$, and traversed by the primary flux $\phi_{P}$.

- $R_{S}$ : Reluctance of the secondary circuit, length $\ell_{S}=6,5 . a$, section $S_{2}$, and traversed by the primary flux $\phi_{S}$.

- $R_{1 S h}$ : Reluctance of one shunt of the usual TDMS, length $\ell_{1 S h}=2,5 . a$, section $S_{1 S h}=h . b$, and traversed by the flux $\phi_{1 S h}$.

- $R_{2 S h}:$ Reluctance of the equivalent shunt of the TSMS, length $\ell_{2 S h}$, section $S_{2 S h}$, and traversed by the primary flux $\phi_{2 S h}$. Such as: $R_{2 S h}=\frac{1}{2} \cdot R_{1 S h}$ and $\phi_{2 S h}=2 \cdot \phi_{1 S h}$.

The new geometrical construction parameters of the equivalent TSMS are presented in figure. 4. In the rest of this study, we keep for the usual TDMS and its equivalent TSMS :

- The same primary and secondary winding turns. 
- The same distribution if instantaneous flux on the primary $\phi_{P}$, secondary $\phi_{S}$ and shunt side :

$$
\phi_{2 S h}=2 . \phi_{1 S h}
$$

So, to keep the same primary and secondary magnetic inductions, we must keep the same sections :

$$
S_{1}^{\prime}=S_{1} \text { and } S^{\prime}{ }_{2}=S_{2}
$$

Then:

$$
\text { 2.a.b }=a^{\prime} \cdot b^{\prime}
$$

- The same magnetic state in primary $\mu_{P}$, secondary $\mu_{s}$ and shunt side:

$$
\mu_{2 S h}=\mu_{1 S h}
$$

So, the relation: $R_{2 S h}=\frac{1}{2} \cdot R_{1 S h}$ gives:

$$
\frac{\ell_{2 S h}}{\mu_{2 S h} \cdot S_{2 S h}}=\frac{1}{2} \cdot \frac{\ell_{1 S h}}{\mu_{1 S h} \cdot S_{1 S h}} \text {. }
$$

This leads automatically to two possible solutions for geometric configurations of the equivalent TSMS :

\section{- First geometric configuration:}

We choose $: S_{2 S h}=S_{1 S h}$ and $\ell_{2 S h}=\frac{1}{2} \cdot \ell_{1 S h}$

With : $\quad B_{2 S h}=2 . B_{1 S h}$ and $H_{2 S h}=2 . H_{1 S h}$

\section{- Second geometric configuration:}

We choose : $S_{2 S h}=2 . S_{1 S h}$ and $\ell_{2 S h}=\ell_{1 S h}$

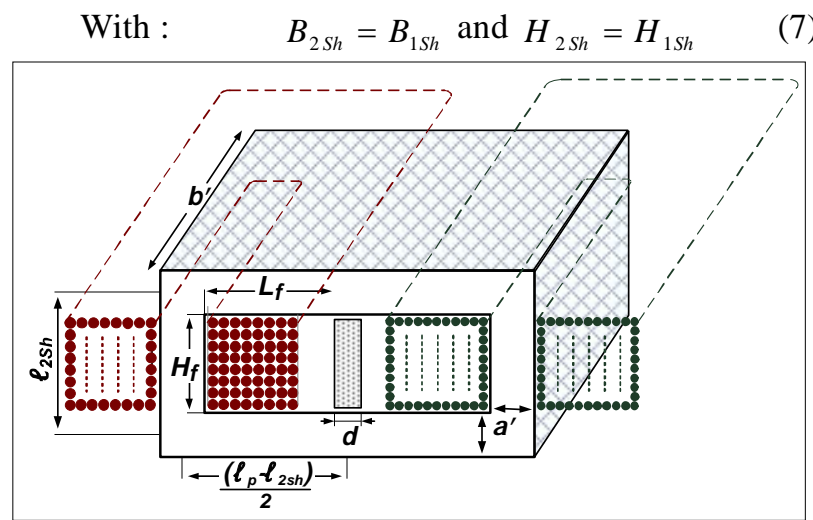

Figure 5 The window of implantation of the primary and secondary

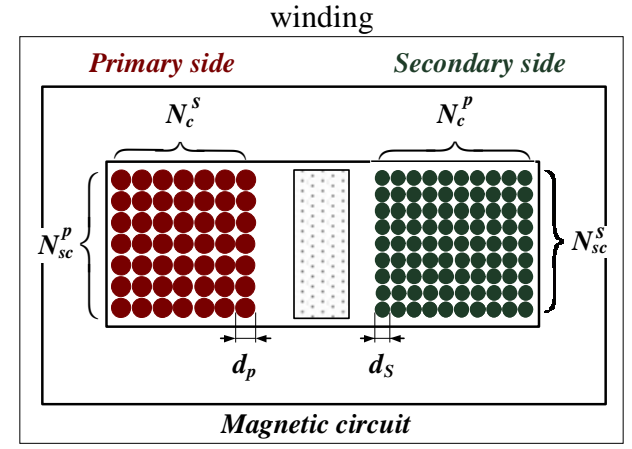

Figure 6: Parameters of the primary and secondary winding

\subsection{Feasibility study of the equivalent TSMS}

Before treating in detail the different geometrical configurations of the equivalent transformer TSMS, we must check their feasibility. Indeed, let us detail the two constraints of realization that the equivalent transformer must respect to be concretely realizable:

\section{A. First constraint:}

From Figure 5, it is clear that the equivalent shunt length $\ell_{2 S h}$ must be strictly greater than the double of the length $a^{\prime} / 2: \ell_{2 S h} \succ 2 .\left(a^{\prime} / 2\right)$.

The first constraint to be respected by different equivalent configurations of the TSMS is:

$$
a^{\prime} \prec \ell_{2 S h}
$$

The multiplication of the inequality (8) by $b^{\prime}$ gives

$$
a^{\prime} . b^{\prime} \prec \ell_{2 S h} . b^{\prime}
$$

According to the relation (2), we can write: $2 . a \cdot b \prec \ell_{2 S h} \cdot b^{\prime}$

This provides:

$$
\rightarrow b \succ\left(\frac{2 . a}{\ell_{2 S h}}\right) \cdot b
$$

The first constraint is simple to verify, but it presents infinity of solutions for each geometric configuration of the equivalent TSMS. Among all the possible geometries obtained, we will look for the solutions that also verify the following second constraint of realization:

\section{B. Second constraint:}

The magnetic circuit window of the feasible geometrical configuration must be sufficient to accommodate all the primary and secondary windings turns.

Figure. 5 show the parameters of the magnetic circuit window and the figure. 6 show the primary and secondary winding parameters. We define:

- $d_{P}, d_{S}$ : Diameter of the primary and secondary winding wire, such as : $d_{P}=1,5 \mathrm{~mm}, d_{S}=0,46 \mathrm{~mm}$.

- $\quad N_{S C}^{P}, N_{S C}^{S}$ : Number of turns per column of height $H_{f}$, for the primary and secondary winding.

- $\quad N_{C}^{P}, N_{C}^{S}$ : Number of the primary and secondary winding columns

- $\quad \ell_{m}^{P}, \ell_{m}^{S}$ : Minimum widths required to accommodate the number of primary and secondary winding columns 
Let us first determine the dimensions of the magnetic circuit window of figure. 5:

- The height: $H_{f}=\ell_{2 S h}-a^{\prime}$

- The width : $\quad L_{f}=\frac{1}{2} \cdot\left(6,5 \cdot a-a^{\prime}-\ell_{2 S h}-d\right)$

From Figure. 5 and 6, we can calculate:

$$
\text { - at primary: } \begin{aligned}
& N_{S C}^{P}=E\left(\frac{H_{f}}{d_{P}}\right)=E\left(\frac{\ell_{2 S h}-a^{\prime}}{d_{P}}\right) \\
& N_{C}^{P}=\frac{n_{1}}{N_{S C}^{P}} \\
& \ell_{m}^{P}=N_{C}^{P} \cdot d_{P}=\frac{n_{1} \cdot d_{P}}{N_{S C}^{P}}
\end{aligned}
$$

$\mathrm{E}(\mathrm{x})$ is the function of the integer part of the real number $\mathrm{x}$

$$
\begin{aligned}
\text { - at secondary : } & N_{S C}^{S}=E\left(\frac{\ell_{2 S h}-a^{\prime}}{d_{S}}\right) \\
N_{C}^{S} & =\frac{n_{2}}{N_{S C}^{S}} \\
\ell_{m}^{S} & =N_{C}^{S} \cdot d_{S}=\frac{n_{2} \cdot d_{S}}{N_{S C}^{S}}
\end{aligned}
$$

Thus, the second constraint consists of having the width $L_{f}$ of the winding implantation window strictly greater than the minimum widths $\ell_{m}^{P}$ and $\ell_{m}^{S}$ necessary to accommodate the number of the primary and secondary windings columns. So we can write:

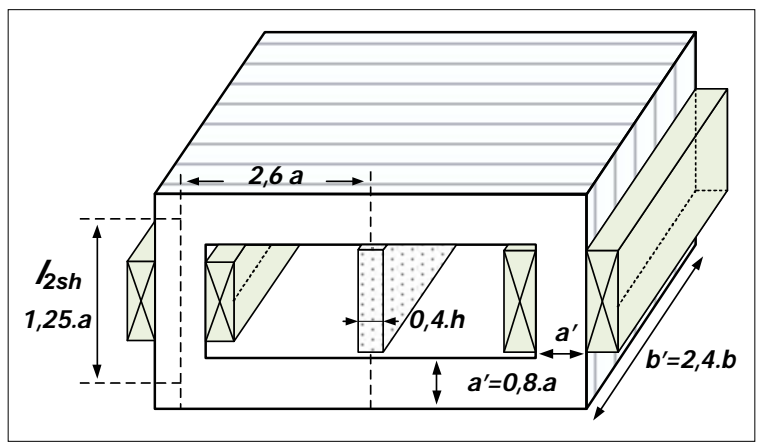

Figure 7 : Geometry of the first configuration of TSMS for $b^{\prime}=2,4 . b$.

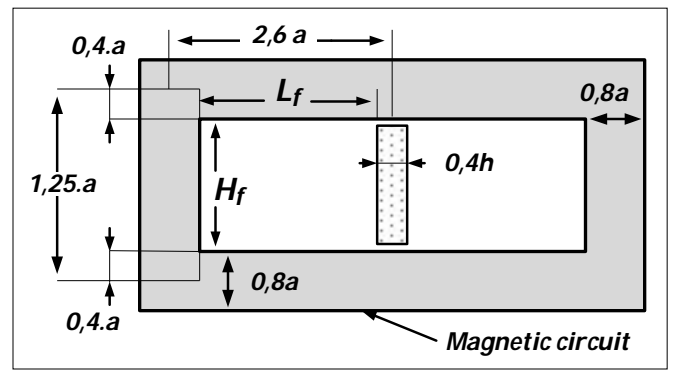

Figure 8 : Geometry of magnetic circuit window for the first configuration of TSMS for $b^{\prime}=2,4 . b$.
- at primary: $\quad L_{f} \succ \ell_{m}^{P}$

So: $\quad L_{f} \cdot N_{S C}^{P} \succ n_{1} \cdot d_{P}$

With: $\quad n_{1} \cdot d_{P}=336 \mathrm{~mm}$

- at secondary: $\quad L_{f} \succ \ell_{m}^{s}$

So: $\quad L_{f} \cdot N_{S C}^{S} \succ n_{2} \cdot d_{S}$

With: $\quad n_{2} \cdot d_{s}=1104 \mathrm{~mm}$

The application of this last constraint is complicated in the general case. We then seek, by manual calculation, the approximate value the TSMS's geometric parameters which satisfy the second constraint. Thus, we can confirm that the geometric configuration is feasible.

\subsection{Sizing of feasible geometric configurations of the TSMS}

We will then develop the two possible geometrical configurations of the equivalent transformer obtained in paragraph 2.1.

\section{A. First Configuration:}

The first geometrical configuration oh the equivalent TSMS resulting from relation (4) gives:

$$
\begin{aligned}
& \ell_{2 S h}=1,25 \cdot a \\
& d \cdot b^{\prime}=h \cdot b
\end{aligned}
$$

We must first determine the magnetic circuit width $b^{\prime}$ of the equivalent transformer taking into account the constraints of realization. After, we can calculate the construction parameters:

- $a^{\prime}$ : the wound core width using the relation (2)

- $d$ : the width of the equivalent shunt's stacking sheets by means of the equality (17).

The results of calculations of the parameters of feasible solutions for the first configuration indicate that:

- For an approximate value of $b^{\prime} \prec 2,4 . b$, the relations (14) and (15) of the second constraint are not verified, which corresponds to geometrical configurations not achievable.

- For an approximate value of $b^{\prime} \geq 2,4 . b$, the second constraint is verified, which gives feasible solutions of the first geometric configuration.

We chose to present the first equivalent geometric configuration of the equivalent transformer (TSMS) for the case of $b^{\prime}=2,4 . b$, taking into account the verification of the constraints of realization. 
Figure. 7 shows the size values of the first geometric configuration of the equivalent TSMS for the case: $b^{\prime}=2,4 . b$, according to the TDMS construction parameters.

By applying relations (2), (16) and (17), we conclude different dimensions obtained:

- The wound core width: $a^{\prime}=0,83 \cdot a$

- The magnetic circuit width: $b^{\prime}=2,4 . b$

- The length of equivalent shunt $\ell_{2 S h}=1,25 . a$

- The width of the equivalent shunt's stacking sheets : $d=0,41 . h$

We find that the two inequalities (8) and (9) of the first constraint of realization are verified. Then, we apply relations (10), (11), (12) and (13) to calculate, with the help of figures.7 and 8 , the following parameters of primary and secondary winding window:

$$
\begin{aligned}
& H_{f}=0,42 \cdot a=10,5 \mathrm{~mm} \\
& L_{f}=2,21 \cdot a-0.207 \cdot h=53,3 \mathrm{~mm} \\
& N_{S C}^{P}=E\left(\frac{0,42 \cdot a}{d_{P}}\right)=7 \\
& N_{S C}^{S}=E\left(\frac{0,42 \cdot a}{d_{S}}\right)=22
\end{aligned}
$$

We can then justify the two inequalities (14) and (15) resulting from the second constraint of realization.

$$
L_{f} \cdot N_{S C}^{P} \succ n_{1} \cdot d_{P}
$$

and

$$
L_{f} \cdot N_{S C}^{S} \succ n_{2} \cdot d_{S}
$$

The results found confirm that the first geometrical configuration of the equivalent transformer with a single magnetic shunt is feasible.

\section{B. Second configuration :}

The second geometrical configuration of the equivalent TSMS resulting from the relation (6) gives:

$$
\ell_{2 S h}=2,5 . a
$$

$$
d \cdot b^{\prime}=2 \cdot h \cdot b
$$

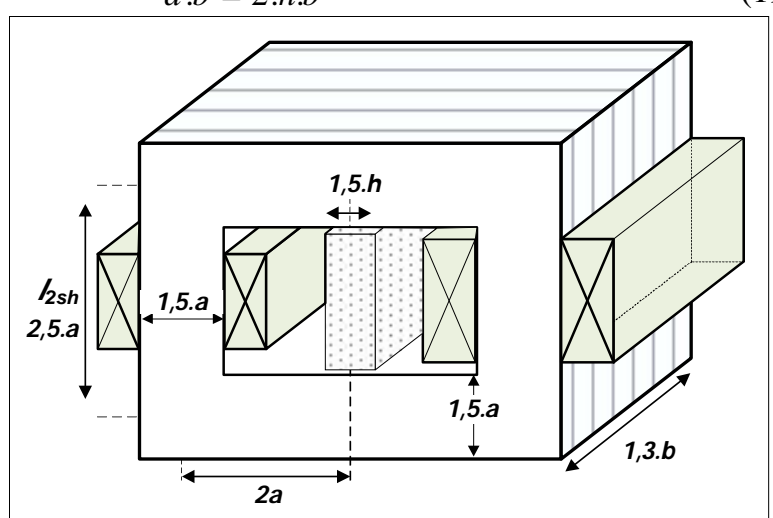

Figure 9 : Geometry of the second configuration of TSMS for

$$
b^{\prime}=1,3 \cdot b \text {. }
$$

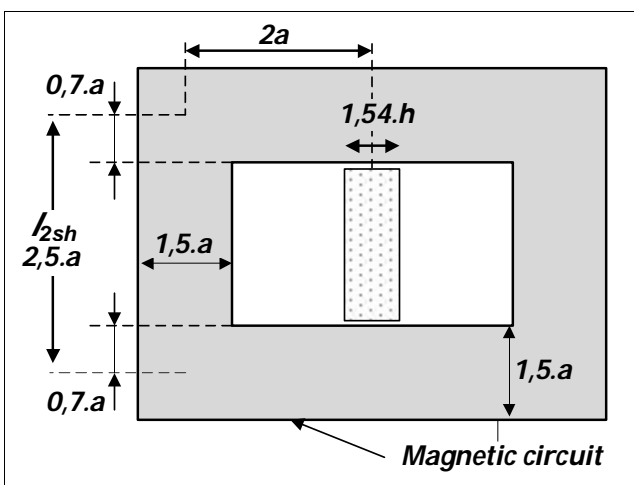

Figure 10 : Geometry of the second configuration of TSMS for $b^{\prime}=1,3 \cdot b$.

As well as the first configuration, we can calculate the construction parameters $a$ and $d$ after finding the value of $b^{\prime}$. Then, the calculation of the parameters of feasible solutions for the second configuration allows concluding that:

- For an approximate value of $b^{\prime} \prec 1,3 . b$, the second constraint is not verified. Then, the corresponding geometrical configurations are not achievable.

- For an approximate value of $b^{\prime} \geq 1,3 . b$, the second constraint is verified, which gives feasible solutions of the first geometric configuration.

We choose to present the calculation obtained from the second equivalent geometric configuration, for the case: $b^{\prime}=1,3 . b$, taking into account the verification of the constraints of realization. Figure. 9 presents the numerical construction values of this configuration. By applying relations (2), (18) and (19), we define :

- The wound core width: $a^{\prime}=1,54 . a$

- The magnetic circuit width: $b^{\prime}=1,3 . b$

- The length of equivalent shunt $\ell_{2 S h}=2,5 . a$

- The width of the equivalent shunt's stacking sheets :

$$
d=1,54 . h
$$

From the figures. 9 and 10, we follow the same procedure of the first geometric configuration, to calculate the following parameters for the second configuration:

$$
\begin{aligned}
& H_{f}=0,96 \cdot a=24 \mathrm{~mm} \\
& L_{f}=1.23 \cdot a-0.77 \cdot h=23.82 \mathrm{~mm} \\
& N_{S C}^{P}=E\left(\frac{0,96 \cdot a}{d_{P}}\right)=16 \\
& N_{S C}^{S}=E\left(\frac{0,96 \cdot a}{d_{S}}\right)=52
\end{aligned}
$$


This makes it possible to check the second constraint of realization for this second geometric configuration.

$$
L_{f} \cdot N_{S C}^{P}=381,1 \mathrm{~mm}
$$

and

$$
L_{f} . N_{S C}^{S}=1238,6 \mathrm{~mm}
$$

In the same way, we can conclude that the second geometric configuration of the equivalent transformer TSMS is realizable.

\section{MODELING AND SIMULATION OF THE TSMS}

\subsection{Equivalent electric model of the TSMS}

The mathematical development of the electrical and magnetic equations of the usual TDMS and its equivalent TSMS leads to the electric model in the figure.11. It is a model of quadrupole in $\pi$, composed of three nonlinear inductances. Each of them is characterized by its non-linear flux-current relationship related to the geometrical parameters of a specific part of the magnetic circuit of the transformer (Primary, secondary, equivalent shunt) [1-17-18-19].

With:

- $u_{1}^{\prime}$ : Voltage $u_{1}$ referred to the secondary circuit.

- $i_{1}^{\prime}$ : Current $i_{1}$ referred to the secondary circuit.

- $i_{P}$ : Current flowing in the primary circuit. $i_{P}{ }_{P}:$ is current $i_{P}$ referred to the secondary circuit.

- $i_{S}$ : Current flowing in the secondary circuit $\phi_{S}$.

- $i_{2 S h}$ : Current flowing in the equivalent shunt of the TSMS. $i_{2 S h}^{\prime}$ is current $i_{2 S h}$ referred to the secondary circuit.

- $r_{1}^{\prime}$ : Resistance $r_{1}$ referred to the secondary circuit.

- $L_{P}$ : Inductance of the primary circuit. $L_{P}{ }_{P}$ is Inductance $L_{P}$ referred to the secondary.

- $L_{S}$ : Inductance of the secondary circuit.

- $L_{2 S h}$ : Inductance of the equivalent shunt of the TSMS. $L_{2 S h}^{\prime}$ is inductance $L_{2 S h}$ referred to the secondary

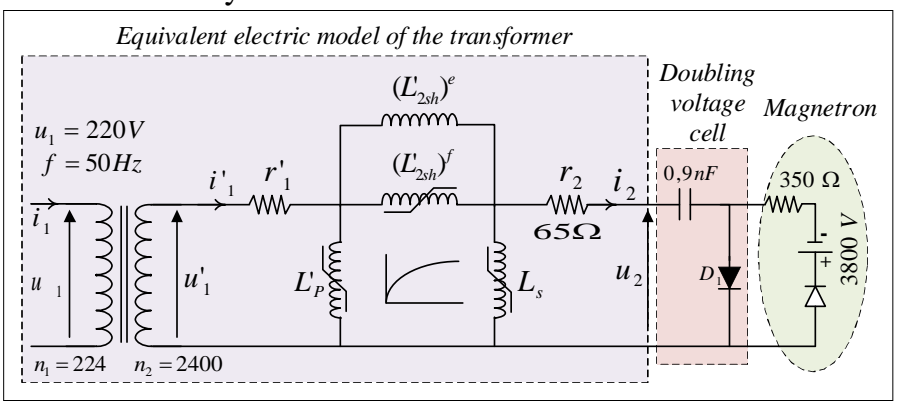

Figure 11: Equivalent electrical model of the power supply using the TSMS for a magnetron
The inductances $L_{P}^{\prime}$ and $L_{S}$ are respectively related to the magnetic circuit reluctances on the primary and secondary sides $R_{P}$ and $R_{S}$. The inductance $L_{2 S h}^{\prime}$ is linked to the reluctance $R_{2 S h}$ of the TSMS shunt, equivalent to the dual identical shunts of the TDMS, having an equivalent air gap of thickness 2.e. The inductance $L_{2 S h}^{\prime}$ is divided into two parallel inductors: the inductance $\left(L_{2 S h}^{\prime}\right)^{f}$ relative to the iron part of the equivalent shunt, and the inductance $\left(L^{\prime}{ }_{2 S h}\right)^{e}$ relative to the gap portion of the same shunt [1-2].

\subsection{Exploitation with EMTP}

The exploitation with EMTP of the diagram of figure.11 consists in implanting in the simulation software the characteristic: $L(i)=\frac{n_{2} \cdot \phi(i)}{i}$ of each nonlinear inductive element [23-24]. We calculate a table giving the total flux $\phi(i)$ for the fictitious current $i$ through each nonlinear inductance. For this, we use the geometric parameters of the TSMS, namely; core width, secondary turns number, gap width, material used-curve $\mathrm{B}(\mathrm{H}) \ldots[21-22]$ relating to primary, secondary and shunt equivalent. For each equivalent configuration of the TSMS treated in paragraph II-2, we determine the expressions of inductances:

\section{A. $\quad L_{P}^{\prime}, L_{S}$ : Primary and secondary inductance:}

Since we keep my same flux distribution in the primary and secondary side, we can find the analytical expression of the primary and secondary inductance for each configuration of the equivalent TSMS. So, we obtain the following expressions of primary and secondary inductances:

$$
\begin{aligned}
& \left(L_{P}^{\prime}\right)\left\{\begin{array}{l}
n_{2} \cdot \phi_{P}=n_{2} \cdot B \cdot S_{1} \\
i^{\prime}{ }_{P}=\frac{\ell_{P}}{n_{2}} \cdot H
\end{array}\right. \\
& \left(L_{S}\right)\left\{\begin{array}{l}
n_{2} \cdot \phi_{S}=n_{2} \cdot B \cdot S_{2} \\
i_{S}=\frac{\ell_{S}}{n_{2}} \cdot H
\end{array}\right.
\end{aligned}
$$

We notice that the expressions of the inductances $L_{P}^{\prime}$ and $L_{S}$ of the new TSMS are the same as those obtained for the usual TDMS [1-2-10]

B. $\left(L_{2 S h}^{\prime}\right)^{f},\left(L_{2 S h}^{\prime}\right)^{e}$ : Inductances of the equivalent shunt

For each geometric configuration of the TSMS, we use the following expressions of the two inductances of the equivalent shunt : 


$$
\begin{aligned}
& \left(L_{2 S h}^{\prime}\right)^{f}\left\{\begin{array}{l}
n_{2} \cdot \phi_{2 S h}=n_{2} \cdot B_{2 S h} \cdot S_{2 S h} \\
i^{\prime}{ }_{2 S h}=\frac{\ell_{2 S h}}{n_{2}} \cdot H_{2 S h}
\end{array}\right. \\
& \left(L_{2 S h}^{\prime}\right)^{e}=\frac{n_{2}^{2}}{\left(R_{2 S h}\right)^{e}}, \text { with }:\left(R_{2 S h}\right)^{e}=\frac{1}{2} \cdot\left(R_{1 S h}\right)^{e}
\end{aligned}
$$

- For the first configuration:

We exploit results (1), (3), (4), and (5) for the first geometric configuration. we get the following expression of $\left(L^{\prime}{ }_{2 S h}\right)^{f}$ :

$\left(L_{2 S h}^{\prime}\right)^{f}\left\{\begin{array}{l}n_{2} \cdot \phi_{2 S h}=n_{2} \cdot\left(2 \cdot B_{1 S h}\right) \cdot\left(S_{1 S h}\right) \\ i_{2 S h}^{\prime}=\frac{\left(\ell_{1 S h} / 2\right)}{n_{2}} \cdot\left(2 \cdot H_{1 S h}\right)\end{array}\right.$

- For the second configuration:

We use relations (1), (3), (6), and (7) for this case, and we obtain;

$\left(L^{\prime}{ }_{2 S h}\right)^{f}\left\{\begin{array}{l}n_{2} \cdot \phi_{2 S h}=n_{2} \cdot\left(B_{1 S h}\right) \cdot\left(2 \cdot S_{1 S h}\right) \\ i^{\prime}{ }_{2 S h}=\frac{\ell_{1 S h}}{n_{2}} \cdot\left(H_{1 S h}\right)\end{array}\right.$

Thus, we get the same expressions of $\left(L_{2 S h}^{\prime}\right)^{f}$ for both configurations of the TSMS [1]:

$\left(L^{\prime}{ }_{2 S h}\right)^{f}\left\{\begin{array}{l}n_{2} \cdot \phi_{2 S h}=2 \cdot n_{2} \cdot B \cdot S_{1 S h} \\ i^{\prime}{ }_{2 S h}=\frac{\ell_{1 S h}}{n_{2}} \cdot H\end{array}\right.$

$\left(L_{2 S h}^{\prime}\right)^{e}=n_{2}^{2} \cdot \frac{2}{\left(R_{1 S h}\right)^{e}}=n_{2}^{2} \frac{2 \cdot e}{\mu_{0} \cdot S_{1 S h}}$

( $\mu_{0}$ is permeability of vacuum)

The equations (20), (21), (22) and (23) will allowed us to exploit with EMTP the power supply model using the new TSMS developed in paragraph II.

\subsection{Simulation and validation of the model}

The simulation with EMTP of the circuit of Figure. 11 was performed during the nominal operation of a microwave generator $800 \mathrm{~W}-2450 \mathrm{MHz}$ [25-26-27]. The characteristics of the power supply using the single-phase shunt transformer studied are [12-16-19] :

- A Transformer characterized by: $f=50 \mathrm{~Hz}$, $u_{1}=220 \mathrm{~V}, u_{2}=2300 \mathrm{~V}, r_{1}^{\prime}=100 \Omega, r_{2}=65 \Omega$ $n_{1}=224, n_{2}=2400$.

- A capacitor of capacity $C=0,9 F$ and a high voltage diode (HVD) for the voltage doubler.

- The magnetron tested, of resistance $R=350 \Omega$, operates under a voltage of about $4000 \mathrm{~V}$. According to the technical specifications imposed by the manufacturer of this tube, the magnetron consumes an average current intensity $I_{m}=300 \mathrm{~mA}$ without exceeding its peak current $I_{\text {Peak }} \succ 1,2 \mathrm{~A}$ capable to destroy it and make it out of service.

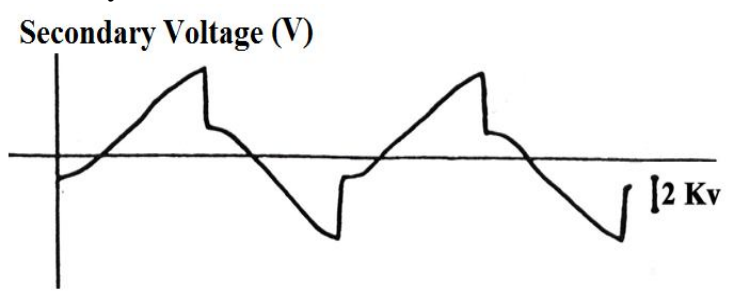

Condenser voltage (V)
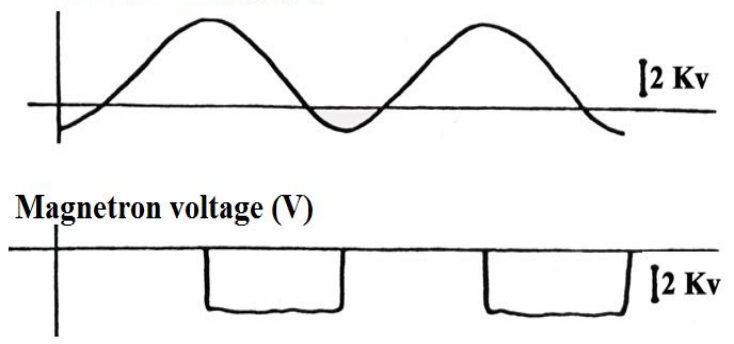

(a) Voltage waveforms
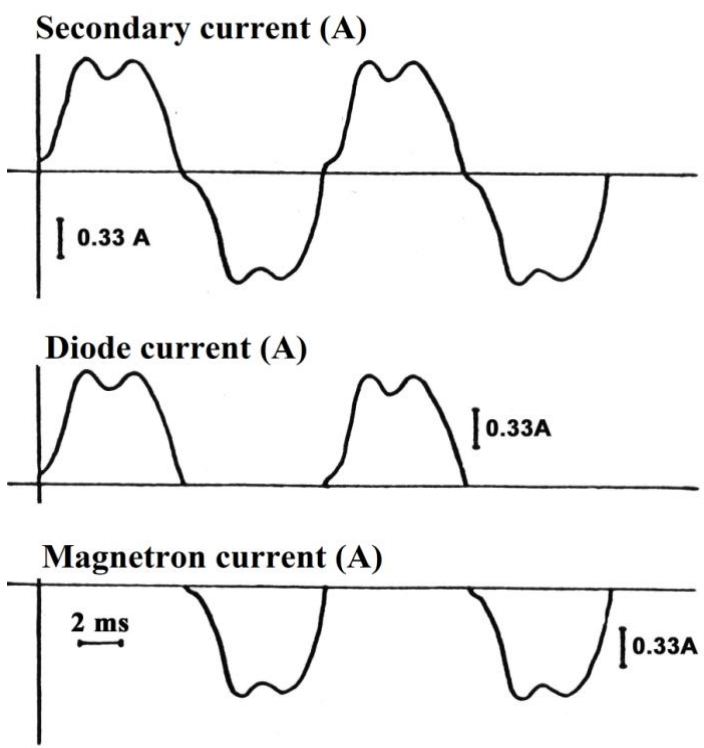

(b) Current waveforms

Figure 12: Results obtained experimentally for the usual TDMS.

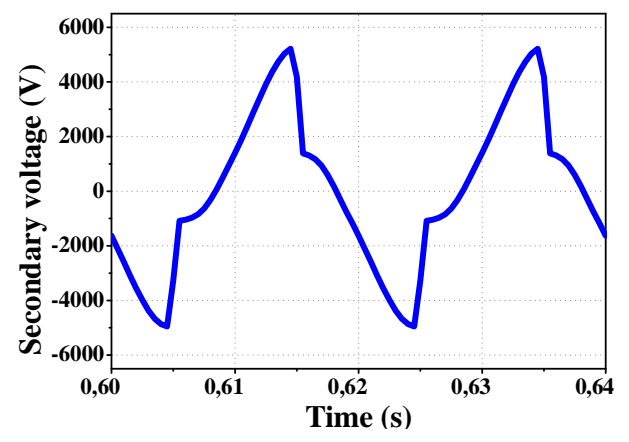



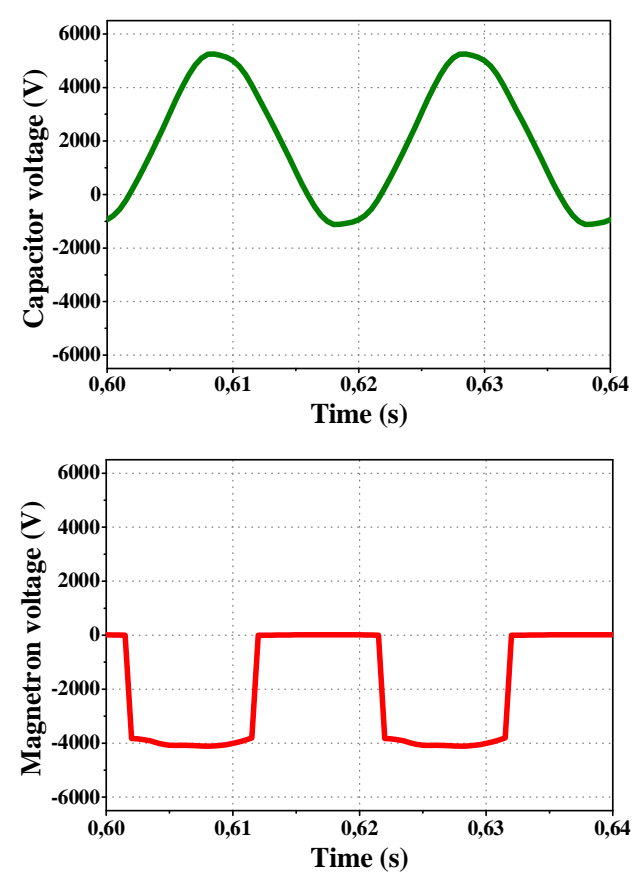

(a) Voltage waveforms
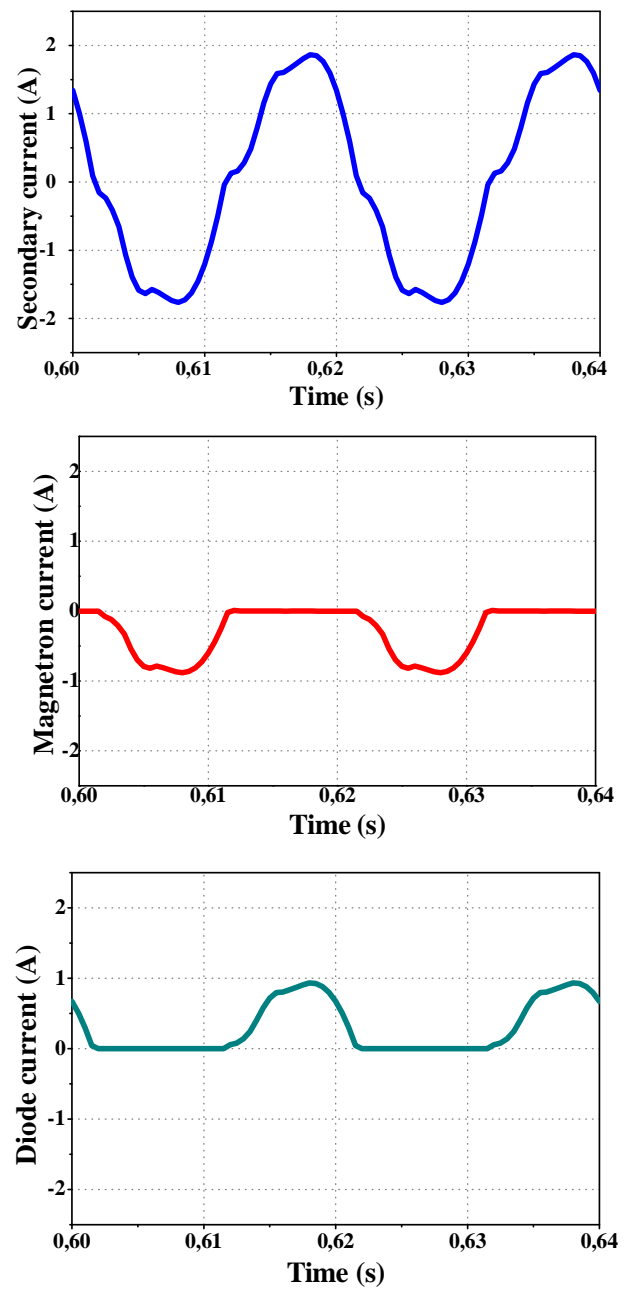

(b) Current waveforms

Figure 13: simulation results for the equivalent TSMS
The simulation results shown in Figure. 13 are compared with the curves of Figure. 12 obtained experimentally in previous tests [2], using a microwave generator $800 \mathrm{~W}-2450 \mathrm{MHz}$ [28]. Indeed, the simulation waveforms of the secondary voltage, capacitor voltage, magnetron voltage of figure. 13-a, are consistent with the voltages found experimentally presented in Figure. 12-a. The secondary current, diode current and magnetron current of figure. 13-b and 12-b are similar for both cases. In addition, the magnetron current complies with the magnetron current regulation conditions ( $I_{m}=300 m A$ and $I_{\text {Peak }} \succ 1,2 A$ ) which confirms the good agreement between the experimental waveforms and the simulation ones.

\section{CONCLUSION}

This article confirms that each geometric configuration of the equivalent TSMS (Tronsformer with a single magnetic shunt) can replace the usual TDMS (Transformer with dual magnetic shunts), in the single-phase HV power supply of a magnetron.

This paper made it possible to find the configuration of a new feasible transformer (TSMS) equivalent to the current TDMS. The different geometric configurations of the TSMS have been correctly dimensioned to ensure the nominal operation of the magnetron thus delivering its full microwave power.

The study of the different geometrical configurations of the TSMS, treated in this article, can represent an addition in the manufacture of the $\mathrm{HV}$ power supplies for industrial microwave generators using single-phase transformers with magnetic shunts. This can be useful in the development of technological innovation in the microwave industry.

\section{REFERENCES}

1. R. Batit. Design and modeling of a new three-phase HV supply for industrial microwave generators with $\mathbf{N}=2$ magnetrons per phase, Ph.D. thesis, ENSAIbnZohr university, Agadir-Morocco, Sept, 2018.

2. M Chraygane, M Ferfra, B Hlimi. Modélisation d'une alimentation haute tension pour générateurs micro-ondes industriels à magnétron. Revue 3EI. 2005; $41: 37-47$.

3. E.G. Dorgelot, Philips Technishe Rundschau, 21934 (1980) 103-109.

4. L. P. Pietta, M. E. Treter, J. S. Barin, and L. Michels, Modelling and control of a high-frequency magnetron power supply for microwave heating applications, IEEE 13th Brazilian Power Electron. Conf. 1st South. Power Electron. Conf. COBEP/SPEC 2016, vol. 2, no. c, 2015.

https://doi.org/10.1109/COBEP.2015.7420098

5. David Greene J., Gross C.A., Nonlinear modelling of transformers, IEEE transactions On Industry Applications, N³, 24 May/June (1988) 
https://doi.org/10.1109/28.2892

6. B. Bahani, N. El Ghazal, M. Chraygane, A. Belhaiba, M. Bousseta, and M. Ferfra, Improved Model of New Six-Phase High Voltage Power Supply for Industrial Microwave Generators with A Single Magnetron By Phase, vol. 4, no. 10, pp. 25-33, 2014.

7. A. Singhal, P. Nijhawan, A. Sinha, K. Rai, Approach for Efficiency Enhancement of Microwave Ovens, Int. J. Ad. Trends. Comput. Sc. Eng, Vol. 8, No.2, March April 2019

https://doi.org/10.30534/ijatcse/2019/27822019

8. M. Bassoui, M. Ferfra, and M. Chraygane, "A A new validation approach of a new three-phase high voltage power supply for microwaves generators with one magnetron by phase," Int. J. Electr. Comput. Eng., vol. 6, no. 1, pp. 1-11, 2016.

https://doi.org/10.11591/ijece.v6i1.8305

9. J. Valainis, Nonlinear Transformer Model for Circuit Simulation, IEEE Trans. Comput. Des. Integr. Circuits Syst., vol. 10, no. 4, pp. 476-482, 1991.

https://doi.org/10.1109/43.75630

10. M. Ferfra et al., Improved modeling of new three-phase high voltage transformer with magnetic shunts, Arch. Electr. Eng., vol. 64, no. 1, pp. 157-172, 2015.

https://doi.org/10.1515/aee-2015-0014

11. A. Belhaiba et al., Comparative Studies of Electrical Functioning of Magnetron Power Supply for One Magnetron, J estr, vol. 6, no. 3, pp. 35-40, 2013.

12. A. Bouzit, M. Chraygane, N. Elghazal, M. Fadel, M. Ferfra, and M. Bassoui. Modeling of new single-phase high voltage power supply for industrial microwave generators for $\mathbf{N}=2$ magnetrons, Int. J. Electr. Comput. Eng., vol. 4, no. 2, pp. 223-230, 2014. https://doi.org/10.11591/ijece.v4i2.5686

13. H. Kako, R. Narita, and T. Nakagawa, Development of compact inverter power supply for microwave oven, IEEE Trans. Consum. Electron. vol. 37, no. 3, pp. 611-616, 1991.

14. A. D. Theocharis. J. Milias-Argitis. Th. Zacharias, Single-phase transformer model including magnetic hysteresis, and eddy currents, Electr Eng vol. 90, 2008, pp. 229-241

15. R. Batit, M. Chraygane, M. Ferfra, M. Fadel, A. Dsoul, and B. Bahani, Breakdowns' diagnosis of a new character three-phase high voltage power supply for industrial microwave generators with $\mathbf{n}=\mathbf{2}$ magnetrons per phase, Int. J. Electr. Comput. Eng., vol. 6, no. 3, pp. 1248-1259, 2016.

16. Jouni Pylvänäinen, Kirsi Nousiainen, Pekka Verho, Studies to Utilize Calculated Condition Information and Measurements for Transformer Condition Assessment, International Review of Electrical
Engineering (I.R.E.E.). Vol.4. n.4. June.2009. pp. 684-689.

17. N. Elghazal, A. Belhaiba, M. Chraygane, and B. Bahani. New Simulation Method of New HV Power Supply for Industrial Microwave Generators with $\mathbf{N}=\mathbf{2}$ Magnetrons, Int. J. Adv. Comput. Sci. Appl., vol. 4, no. 12, pp. 55-64, 2013. https://doi.org/10.14569/IJACSA.2013.041209

18. M. O. Ahmedou, M. Chraygane, and M. Ferfra. New $\pi$ model validation approach to the leakage flux transformer of a high voltage power supply used for magnetron for the industrial micro-waves generators 800 watts, Int. Rev. Electr. Eng., vol. 5, no. 3, pp. 1003-1011, 2010.

19. M. Chraygane, M. Teissier, A. Jammal, J.P. Masson, Modeling of a shunt transformer used in the HV supply of a microwave generators for a magnetron, $J$ de physique III, (1994) 2329-2338.

20. C. STRIMBU, C. G. CONSTANTINESCU, M. PEARSICA, and L. MIRON, Using the Hysteresis Loop to Study a Single-Phase Transformer Working in AC-Switching Regime, Adv. Electr. Comput. Eng., vol. 7, no. 1, pp. 44-49, 2007.

21. Guanghao Liu, Xiao-Bang $\mathrm{Xu}$, Improved modeling of the non-linear B-H Curve and its Application in Power Cable analysis, IEEE Transaction on magnetics, Vol. 38, $\mathrm{N}^{\circ}$.4, July 2002

22. Kawkabani, J.-J. Simond, Improved Modeling of Three-Phase Transformer Analysis Based on Magnetic Equivalent Circuit Diagrams And Taking Into Account Nonlinear B-H Curve, Journal Electromotion, Volume 13, Number 1, January-March 2006, pp. 5-10.

23. Dick E.P., Wastom W., Transformers models for transcient studies based on field measurements. IEEE transactions, PAS-100, Nº1, (1981) 409-419

24. Chan J. H., Vladimirescu A., GAO X. C., Liebmann P, Valainis J., Non linear transformer model for circuit simulation, IEE Transactions on computer-Aided, $\mathrm{N}^{\circ} 4,10$, April (1991)

25. Hermann W. Dommel, ElectroMagnetic Transcients Program, Reference Manual, EMTP Theory Book, 1986

26. Emperreur G., Transformers modelling basic theory, exemples, Leuven EMTP Summer course, Belgium, July 1991.

27. Roguin J., Ranjamina V., modelling of magnetic circuits with EMTP, EDF, bulletin de la DER - serie B, réseaux électriques, matériels électriques, $\mathrm{N}^{\circ} 2$, pp 23-26, 1986

28. S. Kanithi, S. Mahule, A. Gupta, Hybrid Cylindrical Dielectric Resonator Antenna for Microwave Applications, Int. J. Ad. Trends. Comput. Sc. Eng, vol. 7 No.2, March-April 2018 